\title{
Paul Ginsborg
}

\section{Die italienische Krise}

Gegen Ende des Jahres 1991 waren führende christdemokratische und sozialistische Politiker in aller Ruhe damit beschäftigt, die Grundzüge der italienischen Politik im neuen Jahrzehnt festzulegen. Am 17. November 1991 berichtete Repubblica, Craxi und Andreotti hätten ein Abkommen über weitere fünf Jahre einer gemeinsamen Regierung unter Dach und Fach gebracht. Unvorsichtigerweise verkündete am selben Tag der ehemalige Innenminister und Mafiaboß von Neapel, Antonio Gava, daß »wir von nun an Stimmen bekommen aufgrund dessen, was wir darstellen, und nicht bloß wegen unseres Antikommunismus«. Niemand hätte weiter daneben liegen können. Doch damals hätten, wenn überhaupt, nur wenige Beobachter gewagt, ein radikal anderes Szenario vorauszusagen. Die Mängel des italienischen Staates und seines politischen Systems waren längst durchforscht und angeprangert worden, doch Jahr für Jahr bestätigte sich aufs neue die Stabilität der italienischen Wählerschaft und die Zähigkeit der politischen Elite.

Es sollte sich jedoch herausstellen, daß die Jahre 1992 bis 1994 mit zu den dramatischsten im Italien des 20. Jahrhunderts zu zählen sind. Im Verlauf eines einzigen Jahres, 1993, wurden die fünf herrschenden Parteien der Nachkriegszeit von der politischen Landkarte radiert. Eine Gruppe von Richtern und Staatsanwälten ging auf eine in der Geschichte des vereinten Italien beispiellose Weise zum Angriff gegen die Korruption über. Gegen die Mafia wurde ein unnachgiebiger Krieg geführt, der bei der sizilianischen Bevölkerung, besonders in Palermo, zum ersten Mal breite Unterstïtzung fand. Die alte politische Elite, dem Anschein nach so unsterblich wie die japanische, stürzte nicht nur aus ihren Machtpositionen, sie geriet zugleich in übelsten Verruf. Bei den Wahlen vom März 1994 schließlich, dem politischen Höhepunkt der Krise, errang eine ungewöhnliche, von dem Unternehmer Silvio Berlusconi geführte Koalition unter Einschluß der Neofaschisten einen gewaltigen Sieg.

Selbst aus dieser bloßen Aufzählung der wichtigsten Elemente der Krise und ihrer wesentlichen politischen Auswirkungen wird deren komplexe und widersprüchliche Natur sofort ersichtlich. Die Krise 1992-94 ist kein homogener und innerlich zusammenhängender historischer Prozeß wie die europaweiten Entwicklungen von 1968-69. Vielmehr besteht die Krise aus 
sehr verschiedenen Elementen, von denen mehr als eines mit anderen in offenem Widerspruch steht. Im Kern der Krise findet sich keine politische Partei oder Strategie, keine einzelne Klasse oder gesellschaftliche Kraft, die sie verursacht, vorwärtstreibt und ihre Früchte emtet. Unter verschiedenen Gesichtspunkten zeigt sie ganz unterschiedliche Gesichter: vom Mailänder Justizpalast aus handelt es sich um eine Schlacht gegen die Korruption und für die Wiederherstellung der Rechtsstaatlichkeit; aus der Perspektive der Bank von Italien geht es um eine Schuldenkrise und den Verlust des internationalen Vertrauens; für die Lombardei und Venetien geht es um Dezentralisierung, eine neue gesellschaftliche Blockbildung und den Triumph des Selfmademan; im Parlament liegt der Schwerpunkt auf den Wahlgesetzen und dem Abgang der alten Elite; in der zerbrechlichen Zivilgesellschaft Palermos geht es um einen verweifelten Kampf gegen die Macht der Mafia.

Im folgenden wird versucht, die Ursachen dieser außerordentlichen Krise und die Formen, die sie angenommen hat, zu erklären. Zu Beginn mögen einige methodologische Bemerkungen angebracht sein. Erstens hilft es nicht weiter, eine sozusagen kassandrische Sicht der jüngsten italienischen Krise zu vertreten, derzufolge sich die italienische Republik seit ihrer Gründung andauernd in der Krise befand und ihr Niedergang angesichts der vielen zerreißenden Widersprüche unvermeidlich und nur eine Frage der Zeit war. Eugenio Scalfari war immer einer der deutlichsten Vertreter dieser Auffassung. Zwar würden nur die eifrigsten Apologeten der Christdemokraten versuchen, die vielen Risse der Republik wegzuleugnen, doch wenn wir unser Augenmerk allein auf diese Mängel richten, wird es uns nicht gelingen, den Zeitpunkt und die spezifische Form der Krise zu erklären. Vielmehr soll gezeigt werden, daß die Krise 1992-94 im gleichen MaBe den Tugenden der italienischen Demokratie wie ihren Lastern zuzuschreiben ist und in der Tat unverständlich bleibt, wenn nicht beides in Betracht gezogen wird.

Zweitens soll eine monokausale Interpretation der Ereignisse vermieden werden. Staltdessen werden unterschiedliche Sphären - die ökonomische, politische, soziale und kulturelle - betrachtet und verschiedene Ebenen der Erklärung benutzt: lang- und kurzfristige, externe und interne, strukturelle und subjektive.

\section{Staatsverschuldung und internationales Vertrauen}

Nach der großen Wirtschaftskrise 1974-75, in der »die Autoritäten fast die Kontrolle über die Lage verloren « (Giavazzi und Spaventa 1988: 10), konnte die italienische Ökonomie in den darauffolgenden Jahren eine ganze Reihe positiver Indikatoren vorweisen. Das reale BIP stieg zwischen 
1976 und 1990 um fast 50\%, 6\% mehr als der EG-Durschnitt (Padoa Schioppa Kostoris 1993: 225). Der italienische Anteil an den Weltmarktexporten lag im Jahr 1970 bei 4,6\%, fiel 1982 auf 4,4\% und stieg 1987 wieder auf 5\% (Zamagni 1994: 365, Tafel 12.4). Der Inlandsmarkt war in dieser Zeit äußerst lebhaft (der Konsum lag 13 Punkte über dem EGDurchschnitt), und die Sparneigung - wie auch die Konsumneigung - der Bevölkerung lag in der Spitzengruppe der westlichen Länder. Michael Porter wies in seinem Zehn-Länder-Vergleich ökonomischer Wettbewerbsvorteile anerkennend auf die »bemerkenswerte Fähigkeit« der italienischen Industrie hin, innovative Produkte auf den Markt zu bringen und die neuesten Produktionsverfahren und Technologien in relativ kleinen und mittleren Firmen zum Einsatz zu bringen. Sein Lob galt auch den »anspruchsvollen und fortgeschrittenen Inlandsnachfragern und der Entwicklung einer italienischen Zulieferindustrie von Weltrang« (Porter 1989: 691).

Allerdings war auch die Kehrseite der italienischen Wirtschaftsleistung in dieser Periode bemerkenswert. Es handelte sich um die Unfähigkeit, in Forschung und Technologie zu investieren, den Mangel an internationalen Unternehmen in einer Zeit beschleunigter globaler Konzentration (Baldassari 1994: 15) und um die deutliche Schwächung der internationalen Position zwischen 1979 und 1993 im Schlüsselsektor der Güter mit hohen Skalenerträgen: Autos, langlebige Konsumgüter wie Waschmaschinen, elektronische Büroausstattung, organische und anorganische Chemieprodukte usw. (Guerrieri 1994: 384, Tafel 1).

Hinzu kam, daß die italienische Wirtschaftsleistung wegen des ungebändigten Wachstums der öffentlichen Verschuldung international mit wachsender Besorgnis beobachtet wurde. Dies war kein historisch überkommenes Problem der italienischen Republik; im Jahr 1960 etwa hatte Italien eine sehr niedrige Kreditaufnahme und eine geringe Schuldenquote (Giavazzi und Spaventa 1988: 5). Die Lage verschlechterte sich langsam in den siebziger Jahren und dramatisch in den achtziger und frühen neunziger Jahren. Kern des Problems waren die hohen Ausgaben für Renten, beträchtliche aber ineffiziente Kosten des Gesundheitswesens und sehr niedrige Steuereinnahmem von den Selbständigen, insbesonder von Ladeninhabern und den Besitzern von Kleinbetrieben.

In der Zeit der europaweiten Diskussion um den Maastricht-Vertrag, mit deutlicher Neigung zu stärkerer wirtschaftlicher Einheit und einer europäischen Währung, erreichte die öffentliche Verschuldung Italiens Dimensionen eines Staatsnotstands. Im historischen Rückblick hatte Italien in der Europäischen Gemeinschaft eine Rolle eingenommen, die geprägt war durch Passivität und Absentismus, starken verbalen Enthusiasmus und die 
beispiellose Unfähigkeit, EG-Regelungen im eigenen Land durchzusetzen oder auch nur das Geld der Gemeinschaft auszugeben (Giulani 1992).

$\mathrm{Zu}$ diesen schon lange bestehenden Unzulänglichkeiten kam 1991 eine Erkenntnis hinzu, die die Lage noch verschärfte: das Land genügte nicht im entferntesten den drei Mindestbedingungen für die Europäische Währungsunion. Die staatlichen Schulden beliefen sich auf 103\% des BIP (statt maximal 60\%); das Haushaltsdefizit betrug 9,9\%, (statt 4\%) und die Inflationsrate lag bei $6,9 \%$ - weit über dem Durchschnitt der drei leistungsstärksten Länder. Insgesamt gesehen stand nur Griechenland schlechter da (Menet-Gentry 1992: 261-268). Die Folge war eine Art nationale Panik, bei der die Massenmedien wie nie zuvor über die wirtschaftliche Malaise des Landes und seine bevorstehende Relegation aus der ersten Liga der europäischen Nationen berichteten. »Unternehmen Italien, von Schulden erdrückt, verpaßt den europäischen Zug« (La Repubblica, 22. Dezember 1991), lautete nur eine der vielen Schlagzeilen von damals.

Und es sollte noch schlimmer kommen. Im Januar 1990 hatte Giulio Andreotti die italienische Lira in den schmalen Korridor des Europäischen Währungssystems geführt, in der Hoffnung, diese neue Position würde Früchte in Form von Disziplin und Wettbewerbsfähigkeit einbringen. Es stellte sich heraus, daß der hohe Wechselkurs der Lira nicht gehalten werden konnte. Nur zwei Jahre später kam sie unter schweren Druck, und die Regierung von Giulano Amato war zu einer drastischen Intervention gezwungen, um sie zu retten. Schließlich brach im September 1992 das internationale Vertrauen in den Wechselkurs der Lira (und zugleich des britischen Pfunds) völlig zusammen. Kurz danach folgten Abwertung und Austritt aus dem europäischen Währungssystem, schließlich dessen Kollaps.

Die Schuldenkrise und das schwindende internationale Vertrauen können daher nicht einfach als Konstanten der jüngeren italienischen Geschichte behandelt werden. Die gegenwärtige Krise sollte vielmehr als Konflikt zwischen zwei unterschiedlichen Kräften betrachtet werden: Einerseits weigerten sich mehrere italienische Regierungen in Folge, den wachsenden Schuldenberg ernstzunehmen, und beschlossen statt dessen, sich den Konsens im Innern zu erkaufen, mit dem Preis der Schwächung Italiens im internationalen Zusammenhang. In dieser Hinsicht tragen die Regierungen der zweiten Hälfte der achtziger Jahre, einer Zeit des wirtschaftlichen Booms, beträchtliche Verantwortung (eine gute Innenansicht bietet Amato 1990). Andererseits ist festzustellen, daß die fortgeschrittensten und erfolgreichsten kapitalistischen Ökonomien Europas koordiniert in Richtung größerer wirtschaftlicher Einheit, einer gemeinsamen Währung und eines gemeinsamen Marktes vorstoßen. Wiederum handelt es sich nicht um eine Konstante in der Geschichte der Europäischen Gemeinschaft, sondern um eine 
besondere Folge von Ereignissen in den späten achtziger und frühen neunziger Jahren (Tsoukalis 1991).

Diese beiden Entwicklungen, des internationalen Kapitals einerseits und des ökonomischen Mismanagements in Italien andererseits, traten im September 1992 in direkten Konflikt miteinander. Beim ersten Zusammenstoß zog sich das umfassendere europäische Projekt, nicht die nationale leggerezza, mit einer blutigen Nase zurück. Doch der Konflikt der Interessen und Perspektiven wird sich gewiß nicht in Luft auflösen, und auf Iange Sicht sind sehr ungleiche Kräfte daran beteiligt.

In diesem Teil der Erklärung der itaıtıischen Krise spielen externe, strukturelle und langfristige Kräfte die vorherrschende Rolle. Die öffentliche Verschuldung und das internationale Vertrauen sind die »Kontrolleure « der Krise, die Meßlatten, die an alle politischen Lösungsvorhaben angelegt werden.

\section{Tugenden und Laster der italienischen Demokratie}

Die Fehler und Mißerfolge der italienischen Demokratie sind schon häufig, wenn auch mit unterschiedlicher Betonung (Ginsborg 1990, Scoppola 1991, Lanaro 1992, Hine 1993, Lepre 1993), dargestellt worden. Daher genügt hier eine kurze Zusammenfassung. Die in Folge des Verhältniswahlrechts schwachen Regierungen, die jahrzehntelange Vorherrschaft einer Partei und ihrer Verbündeten, deren Inbesitznahme des Staates, das Fehlen einer greifbaren Alternative - all dies hatte schwerwiegende, doch bisher keine tödlichen Folgen für die italienische Demokratie. Die Entwicklung der partitocrazia beschädigte nicht nur die internen Mechanismen des Staates, sondern verlieh auch seinen Interventionen in die Gesellschaft brutale Züge. Die Notwendigkeit, einen Parteisausweis zu besitzen, die (im schlechtesten Sinne) Politisierung der Besetzung aller öffentlichen Ämter (vom Krankenhausverwalter bis zum Opernhausintendanten) und die Aufteilung der Pfrïnde des staatlichen Rundfunks und Fernsehens zwischen den großen Regierungsparteien und der Opposition gehören zu den schlimmsten Merkmalen der italienischen Demokratie.

Ähnliches gilt für den öffentlichen Dienst. Sabino Cassese, ein aufgeklärter und reformorientierter Mann, der auf dem Höhepunkt der Krise Minister für öffentliche Verwaltung war (April 1993 bis März 1994), hat berechnet, daß jeder italienische Bürger 15 bis 20 Arbeitstage im Jahr verliert, weil er sich mit der lähmenden Bürokratie des Landes herumschlagen muß (Presidenza del Consiglio dei Ministri, Dipartimento per la Funzione Pubblica 1993: 13). Im Lauf der Zeit entwickelte sich ein völlig deformiertes Verhältnis von Staat und Bürgern, das auf der Ineffizienz des öffentlichen Dienstes und dessen willkürlichen Ermessensspielräumen beruht. Die 
Geschwindigkeit und Effizienz eines bürokratischen Aktes hängt von dem Druck ab, den ein Bürger auf einen Verwaltungsangehörigen ausüben kann. Selbstredend können nicht alle Bürger den gleichen Druck ausüben. Die Handlungsanreize für die Bürokratie sind dabei unterschiedlicher Gestalt, von der relativ harmlosen Nutzung von Beziehungen bis zur blanken Korruption (Ginsborg 1990: 14).

Diese Laster in der formellen wie informellen Arbeitsweise der italienischen Demokratie sind historisch gewachsen, aber keine unveränderlichen Züge der republikanischen Landschaft. Wie bei der Staatsverschuldung so waren auch hier die achtziger Jahre eine düstere Zeit. Gian Cario Caselii, der oberste Staatsanwalt Palermos nennt sie $\gg$ Jahre der politischen Arroganz, die das Gleichgewicht der Verfassungsordnung bedrohten..., Jahre schamloser Idiotie auf seiten der Machtzentren des Staates« (Casbelli 1993: 15f.). Zusammen mit den Christdemokraten und häufig schlimmer als diese, spielte die Sozialistische Partei Bettino Craxis eine tragende Rolle bei der Systematisierung der korrupten Praxis. Dies war die Zeit, in der Schmiergeldzahlungen für öffentliche Aufträge in hochorganisierter Form abgewickelt wurden und die Finanzierung von Parteien und Personen direkt aus der illegalen Nutzung politischer Handlungsrechte gespeist wurde. Es war mehr im Spiel als der europaweite Niedergang des Interesses an staatlicher Politik, der von vielen Beobachtern vermerkt wurde und seinen Ausdruck in der wachsenden Zahl von Nichtwählern findet (Flickinger, Studlar 1992). Vielmehr schien die Mißwirtschaft der Regierung Teil des Scheiterns des mediteran-romanischen Sozialismus zu sein, denn viele ähnliche Muster staatlicher Beutepolitik fanden (und finden) sich in spanischen, griechischen und selbst französischen Parteien. Anstatt politisches Handeln neu zu erfinden (Dunn 1993: 265) gaben sich die südeuropäischen Sozialisten mit der Maximierung ihrer eigenen Vorteile zufrieden.

Allerdings liegt die politische Erklärung der Krise nicht einfach in den langfristigen, durch die Sozialisten noch verstärkten Defiziten des Systems. Auch die Tugenden der Republik haben eine entscheidende Rolle gespielt. Auf lange Sicht gehörte zu den wichtigsten dieser Elemente die offizielle Moral der in den Jahren 1943-48 geschaffenen Republik. Das italienische Nachkriegsarrangement blieb in vieler Hinsicht hinter den neuen Ordnungen in Frankreich, Westdeutschland und Großbritannien zurück. In Italien gelang es nicht, den Staatsapparat neu zu ordnen oder die Grundlagen für ein gesellschaftsorientiertes Staatsbürgertum zu schaffen. Allerdings waren die Gründungsväter der Republik viel erfolgreicher, als es darum ging, die im Widerstand gegen den Faschismus liegenden Ursprünge der Republik in das institutionelle Gefüge und besonders in die Verfassung einzuarbeiten. Im Blick auf die Nachkriegsjahre läßt sich die Schaffung einer offiziellen Moral nachzeichnen - demokratisch, antifaschistisch, konstitutionell, europäisch -, 
die rhetorisch häufig mißbraucht, doch nie abgelöst wurde. Die Generation der Resistenza endete zum größten Teil an den Rändern der Politik, auch wenn ihre Angehörigen mächtige Zeitzeugen bleiben: Norberto Bobbio, Leo Valiani, Vittorio Foa, Sandro Galante Garrone und, allen voran, weil er wirklich die Macht innehatte und der populärste Präsident der Republik war, Sandro Pertini. In dieser Hinsicht ist der Vergleich mit Japan erhellend, eben weil keine solche Widerstandsgeneration als Gewissen der neuen Demokratie diente (Samuels 1994). In Italien ist eine bestimmte Vorstellung davon, was eine Republik zu sein hat, nie verlorengegangen.

In ännlicher Weise wurde auch die Rechtsstaatlichkeit in zahllosen Fällen mißachtet, aber nie völlig diskreditiert. Sie erlebte in der Nachkriegszeit keineswegs eine Blüte; es genügt, Calamandreis scharfe Kritik der unveränderten Bestände an faschistischen Gesetzestexten zwölf Jahre nach dem Ende des Faschismus zu lesen, um zu verstehen, wie langsam der Wandel vor sich ging (Calamandrei 1955). Doch die Rechtsstaatlichkeit, immer wieder angeschlagen und unterdrückt, kam beharrlich wieder auf die Beine. In der Geschichte der Republik herrscht daher eine beträchtliche Spannung zwischen der alltäglichen politischen Praxis - die häufig auf Begünstigungen, Pöstchenschieberei, Korruption usw. beruht - und der offiziellen Moral, der die Alltagsmoral in letzter Instanz unterworfen ist. Auf symbolischer Ebene wird dies am besten durch den Vorhang von Mario Chiesa verdeutlicht. Chiesa, Direktor des Pio Albergo Trivulzio, war der erste Sozialist, der beim Kassieren von Schmiergeldern erwischt wurde. Seine Verhaftung am 17. Februar 1992 markiert den Beginn des Tangentopoli und wird häufig als offizieller Auslöser der Krise betrachtet. Später sagte er vor Staatsanwalt Di Pietro aus, er habe den Vorhang zugezogen, als die örtlichen Auftragnehmer kamen, um ihn zu bezahlen, damit niemand sehen konnte, was vor sich ging (Della Porta 1993). Er konnte diesen Geschäften nicht am hellichten Tag nachgehen; sie waren immer noch mit einem starken Gefühl für die Unrichtigkeit des eigenen Tuns verbunden.

Diese Spannung zwischen Alltagspraxis und offizieller Moral sollte schließlich zum Zusammenbruch der politischen Klassc Italicns führcn: sic war ihr schwächstes Moment, der Mechanismus, der dem König die Kleider vom Leibe riß. Die politische Klasse brach nicht wegen der Stärke der Opposition, wegen Großdemonstrationen oder hartnäckiger sozialer Bewegungen zusammen. Der Grund dafür war vielmehr, daß sie keine Antwort auf die Anklagen wußte, die, in die Begriffe der offiziellen Moral gekleidet, die Rache eben dieser Moral darstellten. In einem Land, in dem die Ehre immer noch einiges zählt, wog es tatsächlich schwer, auf diese Weise diskreditiert zu werden.

Eine weitere, jüngere Tugend der Republik war der politische Mechanis- 
mus des »Abschaffungsreferendums « (mit dem Gesetze nicht vorgeschlagen, sondern nur bestehende Gesetz abgeschafft werden können). Ursprünglich in der Verfassung verankert, wurde das Recht, Referenden einzuleiten im Mai 1970 Gesetz. 500.000 Bürger, fünf Regionalräte oder ein Fünftel einer der beiden parlamentarischen Kammern haben das Recht, ein solches.Verfahren in Gang zu bringen. Die Debatte über den demokratischen Nutzen von Referenden ist nicht abgeschlossen, und natürlich besteht immer die Gefahr, daß die an der Macht befindlichen Parteien das Verfahren in plebiszitärer Form als populistische Stütze ihrer weitergehenden Bestrebungen einsetzen.

Allerdings war dies in der bisherigen Geschichte der Republik nicht die vorherrschende Rolle des Referendums. Zwar griff Craxi damit recht wirksam die Richterschaft an (siehe unten), doch zumeist waren die Referendumskampagnen soziale Bewegungen mit breiter Basis, bei denen fortschrittliche Gesetze durch die Zivilgesellschaft in Schutz genommen wurden. Dies gilt für die bekannten Referenden zum Scheidungsrecht von 1974 und zum Abtreibungsrecht von 1979; beide waren von seiten des Klerus in Gang gebracht worden und wurden abschlägig beschieden.

Das Referendumsrecht wurde von Mario Segni am 9. Juni 1991 auf neue und dramatische Weise ins Feld geführt. Segni, ein aufrechter, wenn auch nur begrenzt talentierter christdemokratischer Politiker und Sohn eines früheren Präsidenten der Republik, stand bei vielen führenden Mitgliedern seiner eigenen Partei in Ungnade und hatte bei den Sozialisten einen schlechten Ruf. Er stellte mit einiger Unterstützung durch die PDS eine mutige, relativ isolierte Referendumsbewegung für die Abschaffung des »Multiple-Choice« Wahlverfahrens (bei der jeder Wähler mehrere Kandidaten wählen kann) auf die Beine, einem der wesentlichen Mechanismen des politischen Klientelismus. Trotz massiver Angriffe der Medien, trotz Bettino Craxis Ratschlag, gar nicht erst wählen zu gehen, sondern den Tag am Strand zu verbringen, stimmte eine breite Mehrheit der Italiener für Segnis Vorschlag. Dies war der erste schwere Schlag für die herrschenden Parteien und der erste Sprengsatz der Krise.

\section{Die Rolle der Richter und Staatsanwälte}

Der italienische Staat gleicht einem Archipel (Donolo 1980), und darin stellt er eine extreme Variante des Erscheinungsbilds jedes modernen Staates dar: keine zusammenhängende Einheit mit klaren Autoritätslinien, sondern ein Aggregat verschiedener Machtzentren, die in Konflikt miteinander stehen. Im italienischen Fall hat die partitocrazia die vielen Inseln des Staatsarchipels im Laufe der Zeit weitgehend ihrer Kontrolle unterworfen. Allerdings, und dies ist der entscheidende Punkt, diese Kontrolle ist nicht allumfassend. Innerhalb des Staatsapparates verblieben Gruppen, 
»tugendhafte Minderheiten«, die dem Druck der Parteien widerstanden und nicht völlig abhängig waren. Organisation und Angestellte der Bank von Italien sind das bekannteste Beispiel dafür.

Eine dieser Minderheiten, die für die Erklärung der Krise eine zentrale Rolle spielt, besteht aus den Vertretern der Justiz. Ich beschränke mich auf einige wesentliche Punkte, die für meine Argumentation wichtig sind. Entscheidend war wiederum das Nachkriegsarrangement, denn es sah die Einrichtung eines obersten Richterrates (Consiglio Superiore della Magistratura, CSM) vor und schuf die Voraussetzungen für ein unabhängiges Vorgehen der Staatsanwälte. Neppi Modona (1994: 477) weist darauf hin, daß »zum ersten $\mathrm{Mal}$ in der italienischen Geschichte die vollständige Unabhängigkeit der Richter und der Staatsanwälte von der Kontrolle durch die Exekutive festggeschrieben wurde «: Richter und Staatsanwälte waren von nun an einzig dem Gesetz unterworfen.

Diese Autonomie der Justiz steht in scharfem Gegensatz zu vielen anderen europäischen Demokratien. In Frankreich und Spanien ist die Kontrolle der Regierung über die juristischen Untersuchungsverfahren gang und gäbe und hat zur Folge, daß heikle Verfahren, in die Politiker verwickelt sind, andauernd unter den Tisch gekehrt und übereifrige Staatsanwälte kaltgestellt werden (Jean-Pierre 1993; siehe auch das Interview mit ihm im $O b$ server vom 17. Juli 1994). In Großbritannien verläuft die Kontrolle subtiler, denn allein schon das Rekrutierungsmuster der Richterschaft führt zur Schaffung einer, wie Nelken schreibt, »kleinen Gruppe von Vertretern des Establishments mittleren Alters und mittelmäßigen Geistes«.

Der Weg zur Autonomie der Rechtsprechung in Italien war nicht einfach. Der CSM begann erst ab $1959 \mathrm{zu}$ arbeiten, und erst in den späten sechziger Jahren kam mit der Gründung diverser Vereinigungen von Justizvertretern, die die Vielfalt politischer Positionen in der Gesellschaft ingesamt widerspiegelten, der Kampf um eine unabhängige Rechtsprechung wirklich in Gang. Im Kielwasser der Jahre 1968-69 gingen viele idealistische junge Juraabsolventen in die Rechtsprechung (Dalla Chiesa 1992), und nun wurde mit dem Kampf gegen die Mafia und die »poteri occulti $\ll$ der italienischen Gesellschaft Ernst gemacht. Die reformorientierten Juristen blieben eine Minderheit, und es gab viele andere, die weniger verdienstvolle Ziele ins Auge faßten, etwa die Bank von Italien. Dennoch war nun die Zeit zu Ende, in der die Juristen eine geschlossene konservative Kaste bildeten.

Diese ganze Entwicklung mußte natürlich eine Reaktion seitens der offiziellen und inoffiziellen Machtzentren Italiens provozieren. Die späten achtziger Jahre waren eine kritische Zeit, da es schien, als ob der unkontrollierte Handlungsspielraum der Justiz beschnitten werden sollte. Wie 
schon erwähnt, setzten die Sozialisten 1987 das Referendumsverfahren für den Versuch ein, Richter für ihre Fehler finanziell verantwortlich zu machen; mit diesem populistischen Vorschlag errangen sie einen überwältigenden Sieg. Dann startete Francesco Cossiga in der letzten Zeit seiner Präsidentschaft (1990-92) eine Reihe scharfer Angriffe auf den CSM. Das Tangentopoli kam im entscheidenden Moment in Gang, gerade als es den Anschein hatte, der Präsident und die Exekutive hätten die Rechtsprechung an der Gurgel gepackt. Mit den Enthüllungen von Mario Chiesa, einem weiteren schweren Sprengsatz der Krise, wurde eine lebenswichtige Front für den Gegenangriff eröffnet, die von einigen der geistig unabhängigsten, verantwortungsbewußtesten und mutigsten Staatsanwälte des Landes, dem Inbegriff einer tugendhaften Minderheit, auf fähige Weise genutzt wurde. Allerdings hätten sie keinerlei Fortschritte gemacht, wenn ihr Handeln nicht mit anderen Momenten der strukturellen Krise der politischen Elite zusammengefallen wäre.

\section{Gesellschaftliche Transformationen und politische Präferenzen}

Bis zu diesem Punkt scheint die Struktur der Erklärung recht einfach zu sein und fast die Elemente eines moralischen Lehrstücks zu enthalten: die Defizite der italienischen Politik (unter besonderer Berücksichtigung der anstößigen Figuren, die in den achtziger Jahren dafür verantwortlich waren) werden durch externe Faktoren (insbesondere den Trend zur europäischen Einheit) rücksichtslos entblößt und auf überzeugende Weise durch interne Kräfte an der Wurzel gepackt (die Vergeltung der tugendhaften Minderheiten). Wenn wir die Aufmerksamkeit allerdings vom Staat zur Gesellschaft verlagern, wird das Erklärungsmuster undeutlicher und die Krise gewinnt erst ihre ganze Komplexität.

Um die Krise zu ihrer Krise zu machen und sie zum erfolgreichen Abschluß zu führen, mußten die tugendhaften Minderheiten Bündnisse in Staat und Gesellschaft schließen. Auf sich selbst gestellt waren sie viel zu isoliert, um sich Hoffnungen auf einen Sieg machen zu können. Manche Elemente im Spiel, besonders im Jahr 1993, schienen sie bei diesem Unternehmen zu stützen. Auf staatlicher Ebene bot ihnen der neue Präsident Scalfaro vorsichtig Hilfe an, in deutlichem Gegensatz zu seinem Vorgänger. Die Regierung Ciampi (April 1993 - März 1994) tat dasselbe und vermied insofern die Vertuschungsversuche, die so viel zur Diskreditierung der vorangegangenen Regierung unter Giulano Amato beigetragen hatte. Innerhalb der Gesellschaft blieb die öffentliche Meinung fest auf Seiten der Staatsanwälte und widersetzte sich jedem Versuch, die ins Tangentopoli Verstrickten von der Anklagebank zu holen. 
Die Unterstützung der Öffentlichkeit entwickelte sich jedoch nicht zu einer Art kultureller Revolution, wie es sie 1968-69 gegeben hatte. Tangentopoli blieb vor allem ein Zuschauersport, weit entfernt von der Wirklichkeit des täglichen Lebens. Entscheidend war die Unfähigkeit oder die Weigerung, die von den Staatsanwälten aufgeworfenen Fragen mit den Machtstrukturen und der Kultur der italienischen Gesellschaft in Verbindung zu bringen. Die vielen Interessenverbände dieser Gesellschaft - Rechtsanwälte und Notare, Buchhalter und Krankenhausärzte, Universitätsprofessoren und Journalisten - entschlüpften der Krise meistens ohne einen Kratzer. Die überkommene politische Anthropologie der parentela und clientela, des basso profondo von Tangentopoli, wurde nicht in Frage gestellt. Die italienischen Durchschnittsbürger wurden nie aufgefordert, sich unbequeme Fragen zu ihrem eigenen Verhalten zu stellen, danach nämlich, in welchem Maße die herrschende Kultur des Tangentopoli - des Klientelismus, der Korruption, des Nepotismus, der Steuerhinterziehung usw. - auch ihre eigene Kultur war. Um die Gründe dafür zu verstehen, lohnt es sich, wenn auch nur in ganz groben Zügen, die Veränderungen der italienischen Gesellschaft seit Mitte der siebziger Jahre nachzuzeichnen.

\section{Die Entwicklung im Norden}

In Italien waren die Folgen des Postfordismus für die organisierte Arbeiterklasse besonders dramatisch, weil sie in derart scharfem Gegensatz zur Rolle standen, an die sie sich über einen Großteil der Nachkriegszeit hinweg gewöhnt hatte. Während die Arbeiter noch als Protagonisten einer Vision vom grundlegenden Wandel der italienischen Gesellschaft betrachtet wurden - »die gesellschaftliche Kraft, die heute der wichtigste Motor der Geschichte ist « (Berlinguer 1977) - mußten sie mitansehen, wie sich ihre Reihen lichteten, ihre Ideologie entzaubert und ihre Hochburgen geschleift wurden. Die Folge war, besonders im Norden des Landes, eine massive politische und kulturelle Orientierungslosigkeit.

Mit diesem tiefgreifenden Wandel ging der von Ulrich Beck (1986) als »Individualisierung « bezeichnete Prozeß einher. Das Individuum wird immer stärker mit den Risiken und Entscheidungsnotwendigkeiten einer Gesellschaft konfrontiert, in der Großgruppen und Solidaritäten im Schwinden sind, der Zusammenhalt der Familie stark bedroht ist und die Geschlechterrollen radikal neu definiert werden. Gemeinschaft, so Beck, werde im »Säurebad der Konkurrenz« aufgelöst. Um in den Genuß eines immer breiteren Angebots von Konsumgütern zu kommen, muß das Individuum globale wie persönliche Risiken einer ungeschützten Gesellschaft eingehen. Indem es um Bildungsqualifikationen kämpft und 
bereit ist, im dominanten tertiären Sektor des Arbeitsmarkts unbegrenzt mobil und flexibel zu sein, hofft das Individuum, in einer Welt, in der Arbeit immer knapper wird, konstante Beschäftigungsverhältnisse aufbauen zu können. Die Alternative besteht darin, in die wachsende Unterklasse der Unter- und Nichtbeschäftigten abzugleiten, ohne Ersparnisse oder Sicherheiten im Alter.

Die Übertragung des Beck'schen Szenarios auf Norditalien ist kompliziert, da es auf manche Bereiche paßt, auf andere wiederum nicht. Die norditalienische Gesellschaft ist gewiß durch den Niedergang der katholischen wie der kommunistischen Subkultur geprägt (Cartocci 1994), durch immer kleinere Familien (in manchen Regionen wie Ligurien mit einer Reproduktionsrate von Null (Ginsborg 1994c)) sowie durch die wachsende Unabhängigkeit der Frauen und deren Präsenz auf dem Arbeitsmarkt. Allerdings ist die Gesellschaft auch durch sehr starke Familienloyalitäten gekennzeichnet (Rosci 1994), durch verhältnismäßig hohe Standards im Gesundheitswesen und den sozialen Diensten sowie durch das Ethos des Aufbaus kleiner Familienbetriebe. Die Waage von Risikobewußtsein und Konsumentscheidung hat sich daher während der letzten zwanzig Jahre eindeutig in Richtung Konsum bewegt: Häuser, Ferien, Autos, Farbfernseher, Videogeräte, Mobiltelefone, Mode: die ganze Spanne des Hedonismus und der Romantik des Konsums im späten 20. Jahrhundert (Campbell 1989). Auf der anderen Seite sind die Risiken durch eine Reihe von Schutzmechanismen in Schach gehalten worden - vor allem durch die Familie, aber auch durch Beschäftigungschancen in entwicklungsstarken Industrieregionen und recht effiziente Lokalregierungen (Trigilia 1984).

Charakteristisch für diese Gesellschaft sind starke lokale Bindungen und die Geringschätzung von Vorschriften, die Arbeitsbedingungen, Steuern und Abgaben regeln, sowie die wachsende Intoleranz gegenüber der Ineffizienz und Willkür der Zentralregierung; dies ist auch eine Gesellschaft mit mehr als nur einem Anflug von Rassismus (gegen Süditaliener und Einwanderer), mit einer Kultur harter Arbeit, der Bereicherung und der Zurschaustellung des gewonnenen Überflusses. Sie hat sich weit von den Vorstellungen der ethischen Aufrichtigkeit und des Dienstes am Staat entfernt, wie sie in Francesco Saviero Borelli, dem Mailänder Hauptankläger, verkörpert sind. Solche Menschen und ihre Kämpfe werden vielleicht respektiert, doch nur von einem weit entfernten Zuschauerstandpunkt aus.

Natürlich wäre es unsinnig, die gesamte norditalienische Gesellschaft mit diesen sozio-ökonomischen und kulturellen Zügen zu identifizieren. Es gibt Teile der Bevölkerung und geographische Gebiete (städtische Peripherien, entindustrialisierte Zonen), wo die Risiken die Wahlmöglichkeiten der Konsumenten bei weitem übersteigen. In anderen Bereichen widerspricht 
die Tendenz zur Bildung von Verbänden (die im Jahrzehnt vor 1993 landesweit um zwei Prozent zunahmen) der These vom Ende der Großgruppen und Solidaritäten. Die organisierte Arbeiterklasse ist gewiß nicht verschwunden, auch nicht die Fabrikräte. Aber diesen Elementen kommt bei weitem keine vorherrschende Rolle zu, und der Zug der modernen Gesellschaft scheint sich von ihnen zu entfernen.

Die Muster der politischen Artikulation der Mehrheit der norditalienischen Gesellschaft wurden aber auch durch die dramatischen Ereignisse um den Fall der Berliner Mauer und die endgültige Diskreditierung der kommunistischen Regime beeinflußt. Die Stabilität der italienischen Wählerschaft hat sich beträchtlich gelockert, auch wenn es offen bleibt, wie genau die Krise des Weltkommunismus zu diesem Prozeß beigetragen hat und ob er auch ohne sie in Gang gekommen wäre. Der Haupteffekt scheint eher die »Befreiung《 des traditionellen christdemokratischen Wählers als die Flucht des kommunistischen gewesen zu sein. In den »weißen« Gebieten der Lombardei und Venetiens, wo die traditionelle katholische Kultur im Niedergang begriffen ist und die Unzufriedenheit mit den lokalen Politikern grassiert, war der Boden für neue politische Kräfte bereitet (Cartocci 1994), die den oben beschriebenen gesellschaftlichen Wandel widerspiegelten.

Die Lega Nord mit ihrer Verbindung von starkem Regionalismus und erfundener ethnischer Identität, mit ihrer Verachtung für den Zentralstaat und der Überbetonung lokaler Interessen, mit ihrer Verteidung von Individualismus, harter Arbeit und marktliberaler Werte, machte in den Wahlen Anfang der neunziger Jahre rasche Fortschritte. Ihre Hochburgen waren eben jene katholischen Regionen mit dynamischen Kleinunternehmen, doch ihr Einfluß blieb nicht auf sie beschränkt. Auf dem Höhepunkt der Expansion im Jahr 1993 konnte ihr Bürgermeisterkandidat für Mailand, Mario Formentini, mühelos die breite und bunte Front schlagen, die sich aus Angehörigen der gebildeten Mittelschicht, katholischen Freiwilligen und traditionellen Linkskräften um Nando Dalla Chiesa gebildet hatte (Stajano 1993).

Für das Verständnis wesentlich ist die Tatsache, daß die Lega Nord nicht die politische Ausdrucksform vieler neuer gesellschaftlicher Entwicklungstrends im Norden war, sondern daß sie in der Kontinuität überkommener Traditionen steht. Sieht man einmal von der ganzen Rhetorik ab, finden sich wenig Belege dafür, daß die Lega jemals die Absicht hatte, mit dem klientelistischen, familiären und parteidominierten Ethos des alten Regimes zu brechen. Die Lega war eine Revolte gegen das alte Regime, doch ihre politische Kultur hing stärker von diesem Regime ab, als sie eingestehen wollte. 
Der Erfolg der Lega, vor allem bei den Parlamentswahlen von 1992, löste den Würgegriff, in dem Craxis Sozialisten die Mailänder Politik eine Zeit lang gehalten hatten. Borrellis Richterkollegium hatte nun den politischen Spielraum, in dem es sich bewegen konnte. Die Elemente der Krise wiederum stärkten die Sache der Lega. In der Reaktion auf die Schuldenkrise versuchten die Regierungen Amato und Ciampi, den Zugriff auf die Einkommen zu verschärfen, indem sie eine Mindeststeuer für Selbständige einführten und die staatlichen Dienstleistungen beschnitten - all dies in einer Zeit, in der die Wirtschaft sich eher im Abschwung befand. Kleinunternehmen wurden mit Steuerkontrollen überzogen, oft zum ersten Mal, Hausbesitzer mit einer verwirrenden Vielfalt von Steuern. Die Wut des Nordens über die Regierung in Rom fand ihr Ventil in Forderungen nach Autonomie, Privatisierung, freiem Markt - und weiteren Stimmen für die Lega. So gewann die Krise eine kräftige Dynamik, freilich ohne daß es einheitliche Zielvorstellungen gegeben hätte.

Einige Zeit später - doch dies liegt außerhalb dieses Artikels - taucht Silvio Berlusconi als beherrschende Figur im Norden auf, die die Stimmen der Lega aufsaugt und sie in ihre Hochburgen in der Provinz zurïckdrängt. Auch Berlusconi stellte jene für die Lega charakteristische Mischung aus ganz Neuem und sehr Altem dar, doch die Elemente seiner Anziehungskraft unterschieden sich von jenen seines Verbündeten und Rivalen im Norden. Er bot den Mittelklassen ein respektableres und stärker vertrauenerweckendes Bild an als der krude und theatralische Bossi. Berlusconi war Ausdruck der Stimmung Mailands, nicht des Hinterlands. Vor allem aber vertrat er einen ganz anderen Begriff von Politik, der nicht auf lokaler Mobilisierung von unten beruhte, sondern auf dem landesweiten Appell über die Massenmedien, die er in der Hand hatte. Die Politik hatte sich in den siebziger und achtziger Jahren von der Piazza und der örtlichen Parteisektion entfernt und ins Fernsehen verlagert, und sie sollte nach Berlusconis Vorstellung aus dem Fernsehen zur Nation zurückströmen. Viel stärker als Bossi war Berlusconi die Verkörperung einer Reihe von Konsumententräumen - der Selfmademan, der in einem familistischen, anständigen und nationalen Rahmen die Individualität gegen den Kollektivismus verteidigen würde.

\section{Die Zivilgesellschaft im Sïden}

Während der vergangenen zwanzig Jahre machte die süditalienische Gesellschaft langfristige Veränderungen durch, die in vielem dem gesellschaftlichen Wandel im Norden ähnelten, besonders was den Privatkonsum anbelangt. Allerdings gibt es auch grundlegende Unterschiede. Im Süden neigt die Waage von Konsumentscheidung und Risiko viel stärker auf die 
Seite des Risikos. Wenn wir uns wegen seiner bedeutenden Rolle im Gesamtzusammenhang der italienischen Krise nur auf Sizilien konzentrieren, stechen bestimmte Schlüsselmerkmale sofort ins Auge. Die Beschäftigungsmöglichkeiten sind viel begrenzter als in der Lombardei und Venetien, und die Arbeitslosenquoten von Jugendlichen und Frauen sind sehr hoch. Im Osten der Insel gibt es eine Reihe dynamischer kleiner Industriebetriebe, doch die industrielle Struktur der Insel bleibt schwach und abhängig von staatlichen Subventionen (Balistreri 1994). Die sozialen Dienste sind äußerst unzulänglich, die staatliche Hilfe nimmt oft die Form von Almosen für Individuen anstatt von kollektiver Vorsorge an (Bocella 1982). Die Lokalregierungen sind außergewöhnlich korrupt und gründen auf den Prinzipien des Klientelismus (Chubb 1981); aus historischen Gründen waren die Klassensolidaritäten stark begrenzt; Mißtrauen bildet das vorherrschende Element zwischenmenschlicher Beziehungen (Gambetta 1991), und die Zivilgesellschaft besteht eher aus isolierten Außenposten denn aus einem komplexen und gefestigten Netzwerk.

Vor diesem Hintergrund versuchen Familien ihre Chancen durch die Aufrechterhaltung einer relativ hohen Geburtenrate zu maximieren, durch starke traditionale Beziehungen paternalistischer Spielart, durch hartnäckig individualistische Strategien, wie es anhand der enormen Bauwelle der achtziger Jahre deutlich wird: An allen Ecken und Enden wurden Häuser ohne Genehmigung gebaut und oft nicht fertiggestellt. Wie Balistreri schreibt, wird das "tufo giallo - das schlechteste Baumaterial, das überhaupt verwendet werden kann - überall eingesetzt, und von weitem scheint es, als ob ein gewaltiger Sandsturm sich damit vergnügt hätte, sinnlose Formen in der urbanen Wüste zu schaffen« (Balistreri 1990: 170).

Eine Gesellschaft ohne Verantwortlichkeit oder wechselseitiges Vertrauen der Bürger findet ihr traditionelles Ventil in der hochriskanten Entscheidung, für die Mafia zu arbeiten. Einerseits bietet die Mafia die Möglichkeit rascher Bereicherung und konsumtiver Befriedigung, ein starkes Zugehörigkeitsgefühl, Prestige und Ehre. Andererseits gibt es die immer wiederkehrenden Gefahren gegenseitiger Vernichtungskämpfe zwischen verschiedenen Banden, intrafamiliärer Konflikte (Loyalität gegenüber der eigenen Familie und einer Mafia-»Familie« fallen nicht immer zusammen) und zunehmender staatlicher Bekämpfungsmaßnahmen.

Während des hier in Frage stehenden Zeitraums war die Mafia eine dynamisch expandierende Industrie, freilich in immer stärkerem Konflikt mit Elementen der Staatsmacht, besonders nach der Ermordung von General Alberto Dalla Chiesa im Jahr 1982. Sizilien trug zur Krise im wesentlichen durch diesen Konflikt zwischen Staat und Mafia bei. Die in den siebziger Jahren geprägte Generation von Staatsanwälten fand einige ihrer fähigsten 
und mutigsten Exponenten in Sizilien. Das Staatsanwaltsgremium in Palermo, das 1987 den Mammutprozeß gegen 456 Mafiosi organisierte, von denen $19 \mathrm{zu}$ lebenslänglicher Haft verurteilt wurden, zahlte den höchstmöglichen Preis für sein Handeln. Seit den Attentaten auf Giovanni Falcone und Paolo Borsellino im Mai und Juli 1992, schrecklichen Sprengsätzen der Krise im wahrsten Sinne des Wortes, konnte die Kollaboration der alten politischen Klasse mit kriminellen Aktivitäten nicht mehr verschleiert werden. Weniger als ein Jahr später (am 27. März 1993) setzte Gian Carlo Caselli, der neue Chefankläger in Palermo, den dienstältesten Politiker der Republik und sechsmaligen Ministerpräsidenten Giulio Andreotti davon in Kenntnis, daß gegen ihn wegen Zusammenarbeit mit der Mafia ermittelt werde.

Der Kampf gegen die Mafia in Sizilien wurde nicht allein von den Staatsanwälten geführt. Hier, und nur hier, war die Justiz in der Lage, ein Bündnis mit bedeutenden Teilen der Bevölkerung zu schmieden, ein aktives Bündnis im Gegensatz zu dem passiven, das im Norden herrschte. Die Morde an Falcone und Borsellino waren zumindest bei einer beachtlichen Minderheit Anlaß zu einer ethischen, gesellschaftlichen und geistigen Revolte. Es schien, als ob das Mißtrauen, die Gewalt und der Individualismus der sizilianischen Gesellschaft einen Punkt erreicht hätten, an dem als Gegenreaktion eine Massenbewegung ins Leben gerufen wurde.

Wenigstens vorübergehend war Robert Putnams geographische und historische Verortung der zivilen Tugenden in Italien (Putnam 1993) auf den Kopf gestellt worden. Während die Lega Nord die Früchte einer im Kern individualistischen, sezessionistischen und sogar rassistischen Politik erntete, glich Sizilien in den Jahren 1992-93 einem Bienenstock: es gab zivilgesellschaftliche Aktionen und Anti-Mafia-Initiativen, studentische Kampagnen, neugegründete Verbände von Ladeninhabern und Unternehmern und, zum Beispiel, die berühmt gewordene Protestaktion, bei der an einem bestimmten Tag jeder Haushalt, der seine Abneigung gegen die Mafia demonsirieren wollte, ein weißes Laken vom Balkon hängen ließ (Abate 1993).

Der wichtigste politische Ausdruck dieser Bewegung, einzigartig im Sizilien der Nachkriegszeit, war die »Rete « von Leoluca Orlando. Analysiert man den Erfolg (und auch den raschen Niedergang) der Rete, ist es wichtig, ihre gesellschaftliche Basis mit der der Lega Nord zu vergleichen. Während sich in der Lega breite Bevölkerngsschichten wiederfanden, wurde die Rete von der hochgebildeten Jugend der neuen sizilianischen Mittelklassen beherrscht. Eine in Iter (4/92: 27f) veröffentlichte Untersuchung zeigte, daß von den 487 Mitgliedern der Bewegung in der untersuchten Gruppe 30,4\% Universitätsabschlüsse und 52,8\% höhere Schulab- 
chung zeigte, daß von den 487 Mitgliedern der Bewegung in der untersuchten Gruppe 30,4\% Universitätsabschlüsse und 52,8\% höhere Schulabschlüsse besaßen. Ihre Erfahrungen mit zivilgesellschaftlichen Organisationen hatten sie in kulturellen Vereinigungen, Kirchengemeinden, pazifistischen Gruppen usw. gewonnen. Angestellte, Studenten, akademische Freiberufler und Lehrer bildeten $71 \%$ der Mitgliedschaft. 55,5\% waren jünger als 35 Jahre und weitere $24,4 \%$ zwischen 35 und 45 .

Dieser jugendlichen und idealistischen Mittelklassenelite gelang es nur sporadisch, sich mit einer breiteren Basis zu verbinden. Mangels eines starken materiellen Elements zur Ergänzung ihres ethisch geprägten Programms hatten sie immer große Schwierigkeiten, wenn es darum ging, eine stabile politische Mehrheit auf der Insel zu schaffen. Subjektive Irrtümer, besonders ihres charismatischen, aber primadonnenhaften Vorsitzenden Leoluca Orlando, machte die Aufgabe noch schwieriger. Zu Orlandos überwältigendem Triumpf im November 1993 trug die Orientierungslosigkeit der Rechten und die Stimmenthaltung der Mafia einiges bei. Als jedoch Berlusconi im Januar 1994 auf das politische Feld trat, stieß er in diesem Teil des Südens, ebenso wie im Norden, auf fruchtbaren Boden. Sein Versprechen, eine Million neuer Arbeitsplätze zu schaffen, fand auf der Insel besonderen Widerhall. Mit den familistischen und konsumistischen Träumen erreichte er über seine Fernsehkanäle ein Massenpublikum, das für sie genauso aufnahmebereit war wie anderswo. Vor allem jedoch vereinigte die Mafia ihre Stimmen auf ihn. Nicht daß sich Berlusconi absichtlich die Mafia ausgesucht hätte - die Mafia allerdings hat sich gewiß ihn ausgesucht. Tiziana Parenti, für Berlusconis eigene Partei im Parlament, war unter den ersten, die die Infiltration der Forza Italia durch die Mafia anprangerten.

\section{Die Unsicherheiten der PDS}

Das letzte Element der Erklärung hat eher die Form einer Nichtpräsenz als einer Präsenz. Angesichts der radikalen Diskreditierung der herrschenden politischen Parteien, besonders der DC und der PSI, hätte man erwarten können, daß die Opposition deren Platz in der Regierung des Landes einnimmt. Daß dies nicht geschehen ist, stellt eine weitere Besonderheit der italienischen Krise dar.

Im Jahr 1989 bewies Achille Occhetto beachtliche Voraussicht, als er den Namen »Kommunistische Partei Italiens « (PCI) in »Demokratische Partei der Linken « (PDS) umwandelte. Einige Monate lang schien sein Vorgehen eine veränderte Strategie und erneuerte Attraktivität anzuzeigen. Allerdings führte die Namensänderung innerhalb der Partei zu starkem Dissens und schließlich zur Spaltung. Occhetto tat sein Bestes, um zu vermitteln, doch 
der Konflikt endete in einer Selbstblockade. Aus dieser Zeit stammt die lähmende Schwäche der PDS in der Krise: ein Maß an innerem Dissens, das den Großteil ihrer Energien verbrauchte und sie daran hinderte, sich mit hinreichender Kraft und Klarheit nach außen zu wenden.

Die Krise, dies muß inzwischen klar sein, wurde nicht durch die Linke in Gang gesetzt. Ihre (toten und lebenden) Helden waren Falcone und Borsellino, Segni und Di Pietro. Dennoch hätte die PDS durchaus vom Krisengetümmel profitieren können, wie es Silvio Berlusconi schließlich gelang. Es gibt drei Gründe, warum dies nicht geschehen ist (Ginsborg 1994e).

Erstens wurde die PDS selbst von der juristischen Offensive bedroht. In den ersten Wochen des Tangentopoli wurde klar, daß die Pariei in die Mißbrauchsmechanismen des Systems verwickelt war. Occhetto hielt eine Rede, in der er die Nation um Entschuldigung für die Mängel seiner früheren Partei bat, doch sie ging nicht mit handfesten Anzeichen dafür einher, daß die Partei die Absicht hatte, mit sich ins Reine zu kommen und keine Angst davor hatte, die Skelette aus der kommunistischen Schublade zu ziehen. Vielmehr schien die Partei eine Defensivstrategie einzuschlagen und darauf zu warten, daß die Staatsanwälte auf eigene Faust etwas und dann hoffentlich nicht allzu viel ausfindig machen würden.

Von der PDS etwas anderes zu verlangen, wäre schwierig gewesen (wenn es zuviele Skelette gegeben hätte, wäre die junge Partei von einem Haufen alter Knochen erdrückt worden). Allerdings wäre dies die einzige Möglichkeit gewesen, den Unterschied zu den herrschenden alten Parteien aufzuzeigen. Im Frühjahr 1992 klar Schiff zu machen, wäre traumatisch und zerrüttend gewesen, hätte die Linke jedoch in eine viel bessere Position für die entscheidenen Wahlen zwei Jahre später gebracht. Wie die Dinge lagen, und dies zeugt von einer verfehlten Strategie, schien die PDS im entscheidenden Moment ein viel stärker kompromittiertes Element des alten Systems gewesen zu sein, als sie es tatsächlich war.

Zweitens gelang es der PDS fast durchgängig nicht, Initiativen zu ergreifen, mit denen die Krise von den Gerichtshöfen und Bildschirmen weg ins Alltagsleben hineingetragen worden wäre. Hier tauchte von neuem ein alter Fehler der PDS auf: eine gewisse Immobilität und ein Mangel an Phantasie im Hinblick auf moderne soziale Bewegungen. Um die vielen geschlossenen Zirkel in der Gesellschaft aufzubrechen, wäre eine Revolte nötig gewesen; seltsamerweise war eine solche Revolte der politischen Kultur der PCI/PDS fremd.

Schließlich war die Linke insgesamt nicht in der Lage, die Anzeichen der Krise richtig zu deuten. Hat man den Vorteil, die Sache nachträglich beurteilen zu können, läßt sich klar ersehen, daß der Höhepunkt der Krise im Frühjahr 1993 erreicht wurde. Im März wurde praktisch täglich eine neue 
wichtige Figur des Establishments durch die sorgfältige Arbeit der Staatsanwälte diskreditiert. Die politische Klasse fiel auseinander, und dies war der Zeitpunkt, auf eine stärkere Rolle in der politischen Führung zu dringen. Die PDS unterließ dies nicht nur, sondern zog die linken Minister Barbera, Berlinguer, Rutelli und Visco - zurück, die der Regierung Ciampi beigetreten waren. Man hätte alles daransetzen müssen, so früh wie möglich Wahlen abzuhalten. Mit jeder Woche, die verging, gewann die Rechte Zeit, ihre Reihen neu zu ordnen; die Krise flaute ab und der richtige Moment wurde nicht genutzt. Zwar schnitt die Linke bei den Lokalwahlen im November/Dezember 1993 gut ab, doch dies war noch bevor Berlusconi seine Kampagne eingeleitet hatte. Als diese in Gang gekommen war, reagierte die PDS nur halbherzig. Bei den nationalen Wahlen im August 1994 präsentierte sich die Rechte erfolgreich als dynamische, neue Kraft in der italienischen Politik, während die Linke alt, statisch, defensiv und ideenlos erschien.

\section{Die Verknüpfung der Fäden}

Zunächst einmal ist hervorzuheben, daß die Ereignisse 1992-94 eine Krise innerhalb einer Demokratie waren. Vergleiche mit den Ereignissen in Osteuropa übergehen den Unterschied zwischen der Krise eines Einparteienstaates und der Krise in einem Staat, in dem es seit fast fünfzig Jahren freie Wahlen gibt. Und es verschwindet auch die Differenz zwischen einem Land mit einer außerordentlich turbulenten und lebhaften Zivilgesellschaft und jenen Ländern, in denen der Zivilgesellschaft keinerlei Raum eingeräumt wurde.

Es ist ebenfalls wichtig zu betonen, daß das Geschehen in Italien keine Revolution ist, jedenfalls nicht in irgend einer sinnvollen Gebrauchsweise dieses Begriffs. Wie Theda Skocpol (1979) sorgfältig dargelegt hat, geht es bei Revolutionen um Massenaktionen, Staatszusammenbrüche und Staatsgründungen. Es geht um die Zerstörung von Anciens Régimes und um den Versuch, grundlegend andere politische und oft auch gesellschaftliche Systeme aufzubauen und nicht nur um die öffentliche Diskreditierung führender politischer Gestalten. Keines dieser Merkmale trifft auf Italien zu. Vielmehr ist eines der immer deutlicher zutage tretenden Elemente die Kontinuität mit der politischen Praxis der Vergangenheit und der Versuch, die Neuerungen und darauffolgenden Traumata der Jahre 1992-94 zu absorbieren und zu exorzieren.

Eben weil Italien bisher eine Demokratie war, macht es Sinn, die Ursprünge der Krise nicht nur in den Mängeln des demokratischen »Regime « zu suchen, sondern auch im Pluralismus, der allein in der Demokratie möglich ist. Die Herausbildung einer unabhängigen Rechtsprechung und 
die Rolle der mutigen und am Gemeinwohl orientierten Untersuchungsrichter, die Existenz einer höchst spezifischen offiziellen Moral, die auf den antifaschistischen Widerstand zurückgeht, die langsame (wenn auch zu langsame, wie Parker (1994) gezeigt hat) Entwicklung des höheren Schulwesens, die Möglichkeit, von oben wie von unten Referenden auf den Weg zu bringen - all dies sind wesentliche Elemente zur Erklärung des Kampfes gegen die opportunistischen Politiker Italiens.

Auch der äußere Kontext, in dem sich die italienische Demokratie bewegt, hat entscheidend zur Verursachung der Krise beigetragen. Zwei Elemente ragen dabei heraus - das eine befreiend, das andere notwendig beschränkend. Der Fall der Berliner Mauer brachte grundlegende Veränderungen in der Politik des Westens und des Ostens mit sich. In Italien scheint das Ende des Ostblocks wesentlich dazu beigetragen zu haben, die Wähler zu ermutigen, zum ersten Mal seit 1948 ihre Wahlentscheidung ohne, wenn auch vage Rücksicht auf den Kalten Krieg zu treffen.

Noch wichtiger jedoch ist die langjährige Mitgliedschaft Italiens in der EG, durch die allen Bürgern das politische Mißmanagement der Volkswirtschaft in der Zeit der Verträge von Maastricht unweigerlich vor Augen geführt wurde. Die Rettungsaktion der atypischen Regierungen Amato und Ciampi, die eher von technischen Experten als von der Parteipolitik beherrscht wurden, brachte zwangsläufig Einschnitte bei den staatlichen Dienstleistungen und höhere Steuern mit sich. Dies trug in einer Zeit des konjunkturellen Niedergangs noch weiter zur Enttäuschung bei und gab Anlaß, auf extremere und zugleich illusionäre Wahlversprechen einzugehen.

Die Wandlungen des internationalen Umfeldes mußten nicht notwendig zum Niedergang der herrschenden politischen Eliten Italiens führen. Allerdings stellten die achtziger Jahre, trotz aller Modernisierungsbehauptungen der Sozialisten, einen weiteren Verfall der ohnehin schon äußerst meritenlosen Führung der öffentlichen Geschäfte dar. Systematisierte Korruption, schwere Verletzungen der Unabhängigkeit der Rechtsprechung, enorme Verantwortungslosigkeit in Fragen der öffentlichen Verschuldung waren nur die wichtigsten Merkmale der Ära Craxi.

Wenn wir uns den unmittelbaren Auslösern der Krise zuwenden, muß man die überwiegend themenorientierte Analyse der vorangegangenen Seiten in eine chronologische Form bringen. Die wichtigsten Katalysatoren der Krise finden sich im Zeitraum vom Sommer 1991 bis zum Sommer 1992. Das Referendum vom Juni 1991 und die nationale Panik wegen Maastricht waren erste Anzeichen; die Krise kam ernsthaft in Gang mit der Verhaftung von Mario Chiesa im Februar 1992 und dem Sieg der Lega Nord bei den Wahlen im April; in jenem Sommer markierten die Morde an Falcone und Borsellino den dramatischen Eintritt des Südens in die Krise; im Sep- 
tember 1992 wurde die Lira abgewertet und Italien trat aus dem Europäischen Währungssystem aus.

Von nun an taumelte das Land in einem endlosen Strom von Verhaftungen und Selbstmorden, begleitet vom Abgang der politischen Parteien. Für einige Monate verflüssigte sich die italienische Geschichte auf dramatische Weise. Die Krise allerdings schlug mehrere Richtungen ein. Sie schien von den Richtern beherrscht zu werden, doch deren Macht war nur strafend, nicht schaffend. Mit der partiellen Ausnahme Siziliens gab es keine wirkliche Verbindung zwischen politischer Opposition, Zivilgesellschaft und den Anklägern der Justiz. Der Versuch einer »demokratischen Restauration«, einer Rückkehr zu Legalität und Verfassungsmäßigkeit, einer ethischen Revolte gegen die Exzesse der achtziger Jahre weitete sich nie zu einer Politik der Massen und einer kulturellen Revolution aus.

Statt dessen scheute eine Mehrheit der italienischen Wähler vor radikalen Veränderungen zurück und folgte den ganz anders gearteten Appellen zunächst von Umberto Bossi und dann von Silvio Berlusconi. Hinter dieser Entscheidung standen viele der langfristigen gesellschaftlichen und ökonomischen Veränderungen, sowie kulturelle Konstanten, die von der dramatischen Erscheinungsform der Krise gewissermaßen maskiert worden waren: der Trend zur Individualisierung, der Niedergang von Solidaritäten in der Arbeiterklasse, die Auffassung von der Familie als begrenzte Gemeinschaft (De Rita 1988), der Widerhall der Konsumentenrevolution, das Mißtrauen gegen den Staat und die Neigung, ihn zu betrügen, das Überleben einer starken paternalistischen Kultur. Vereinfacht formuliert: zwischen der wirklichen Natur der Gesellschaft und deren politischer Ausdrucksform im Norden gab es mehr Übereinstimmungen als zwischen den »tugendhaften Minderheiten« und der breiteren Öffentlichkeit.

Diese Folgerung, wie sie vehement von Marco Revelli (1994) vertreten wird, führt uns zur Frage nach dem Verhältnis von Struktur und Handlung. Wenn wir dem Argument folgen, wonach die strukturellen Transformationen der letzten beiden Jahrzehnte unvermeidlich zum Triumph der Rechtskoalition Berlusconis führten, bleibt wenig oder kein Raum für die Akteure, seien es individuelle oder kollektive.

Deterministische Erklärungen dieser Spielart beruhen auf einem doppelten Fehlschluß, einem soziologischen und einem methodologischen. Zunächst ist der gesellschaftliche und ökonomische Wandel nicht eindimensional, und seine Folgen sind nicht leicht vorauszusagen (wer hat die Lega oder auch die Ereignisse von 1968 vorausgesehen?). In unserem Fall können steigende Bildungsstandards, die zunehmende Unabhängigkeit der Frauen und die Neigung zur Verbandsbildung als Gegenströmungen zu herrschenden Trends betrachtet werden. 
Zweitens entkräftet die Überbetonung der Struktur auf Kosten der Akteure die historische Erklärung, die darauf beruht, daß beide Momente im Kern interdependent sind. Wird dem Akteursbegriff der ihm zukommende Raum gewährt, gewinnen auch Fehler und Erfolge, die Unsicherheiten der PDS und der außerordentlich bewegliche Feldzug des Silvio Berlusconi ihr Eigengewicht. Dann kann die Komplexität der Ursprünge der Krise, die Vielfalt ihrer Ebenen und Akteure vervollständigt werden durch die Darlegung der Vielfalt ihrer möglichen Ergebnisse.

Aus dem Englischen übersetzt von Klaus Fritz

\section{Literatur}

Abate, F. (1983): Capo d'Orlando. Un sogno fatto in Sicilia, Rom und Neapel.

Amato, G. (1990): Due anni al tesoro, Bologna.

Baldassari, M. (ed.) (1994): The Italian Economy: Heaven or Hell?, New York.

Balisteri, G. (1994): Sicilia: la ricerca di una nuova identità, in: Ginsborg (Hg.) (1994b).

Beck, U. (1986): Die Risikogesellschaft, Frankfurt/M.

Berlinguer, E. (1977): Austerità, occasione per trasformare l'Italia, Rom.

Boccella, N. (1982): Il Mezzogiorno sussidiato, Mailand.

Calamandrei, P. (1955): La Costituzione e le eggei per attuarla, in: Mehrere Autoren, Dieci anni dopo, 1945-55, Bari.

Campbell, C. (1987): The Romantic Ethic and the Spirit of Modern Consumerism, Oxford.

Cartocci, R. (1994); Fra Lega e Chiesa, Bologna.

Caselli, G.L. (1993): La cultura della giurisdizione, in: Micro Mega 5, S. 15-18.

Chubb, J. (1982): Patronage, Power and Poverty in Southern Italy, Cambridge.

Dalla Chiesa, N. (1992): Il giudice ragazzino, Turin.

Della Porta, D. (1992): Lo scambio occulto, Bologna.

De Rita, G. (1988): L'impresa-famiglia, in: P. Melograni, L. Scaraffina (Hrsg.), La famiglia italiana dall'Ottocento a oggi, Bari.

Diamanti, I. (1993): La Lega, Rom.

Donolo, C. (1980): Social change and transformation of the state in Italy, in: R. Scase (Hrsg.), The State in Western Europe, London.

Dunn, J. (Hg.) (1992): Democracy, the Unfinished Journey, Oxford.

Ferrara, M. (1991): Le dodici Europe, Bologna.

Flickinger, R., D. Studlar (1992): The disappearing voters? Exploring declining turnout in Western European elections, in: Western European Politics 15, 2, S. 1-16.

Gambetta, D. (1990): Mafia: The Price of Distrust, in: D. Gambetta (Hrsg.), Trust: Making and Breaking Cooperative Relations, Oxford.

Giavazzi, F., L. Spaventa (Eds.) (1980): High Public Debt: the Italian Experience, Cambridge.

Ginsborg, P. (1990): A history of Contemporary Italy, London.

Ginsborg, P. (Hg.) (1994a): Le virtú della Repubblica, Mailand.

Ginsborg, P. (Hg.) (1994b): Stato dell'Italia, Mailand.

Ginsborg, P. (1994c): La famiglia italiana oltre il privato per superare l'isolamente, in: Ginsborg (1994b).

Ginsborg, P. (1994d): L'Italia, L'Europa, il Mediterraneo, in: Ginsborg (1994b).

Ginsborg, P. (1994e): La sinistra, la crisi, la sconfitta, in: Ginsborg (1994b).

Giuliani, M. (1992): Il processo decisionale italiano e le politiche comunitarie, in: Ginsborg (1994b).

Hine, D. (1993): Governing Italy, Oxford.

Jean-Pierre, T. (1993): Come si manipolano i giudici in Francia, in: Micro Mega 5, S. 69-72.

Lanaro, S. (1992): Storia dell'talia repubblicana, Venedig. 
Lepre, A. (1993): Storia della prinera Repubblica, Bologna.

Menet-Genty, J. (1992): L'Économie italienne, Paris.

Neppi Modona, G. (1994): Giustizia e potere politico, in: Ginsborg (1994b).

Padoa Schioppa Kostoris, F. (1993): Italy: the Shletered Economy, Oxford.

Parker, S. (1994): Review of Ginsborg (ed.), Le virtú della Repubblica, in: ASMI Newsletter 25, S. 41-44.

Porter, M. (1989): The Competitive Advantage of Nations, London.

Presidenzia del Consiglio dei Ministri, Dipartimento per la Funzione Pubblica (1993): Rapporto sulle condizioni delle pubbliche amministrazioni, Rom.

Putnam, R. (1993): Making Democracy Work. Civic Traditions in Modern Italy, Princeton.

Revelli, M. (1994): Forza Italia: l'anomalia italiana non è finita, in: Ginsborg (1994b).

Rosci, E. (1994): Le lunghe adolescenze dell'Italia d'oggi, in: Ginsborg (1994b).

Samuels, R. (1994): Comparative paper on Japan and Italy, presented at the Centre for European Studies, Harvard University, at the conference $»$ Reconstructing Italy: sources of pathology and forces for reform«, Februar (unveröffentlicht).

Scalfari, E. (1969): L'autunno della Repubblica, Mailand.

Scoppola, P. (1991): La Repubblica dei partiti, Bologna.

Skocpol, T. (1979): States and Social Revolutions, Cambridge.

Stajano, C. (1993): Il disordine, Turin.

Trigilia, C. (1986): Grandi partiti e piccole imprese, Bologna.

Tsoukalis, L. (1991): The New European Economy, Oxford.

Zamagni, V. (1994): The Economic History of Italy, 1860-1990, Oxford. 